\title{
Podoplanin Expression in Cancer-associated Fibroblasts Predicts Poor Prognosis in Patients with Squamous Cell Carcinoma of the Lung
}

\author{
YOHEI YURUGI ${ }^{1,2}$, MAKOTO WAKAHARA ${ }^{1,2}$, YUKI MATSUOKA $^{1,2}$, TOMOHIKO SAKABE $^{1}$, \\ YASUAKI KUBOUCHI ${ }^{1,2}$, TOMOHIRO HARUKI ${ }^{2}$, KANAE NOSAKA $^{1}$, KEN MIWA $^{2}$, KUNIO ARAKI ${ }^{2}$, \\ YUJI TANIGUCHI ${ }^{2}$, TATSUSHI SHIOMI ${ }^{1}$, HIROSHIGE NAKAMURA ${ }^{2}$ and YOSHIHISA UMEKITA ${ }^{1}$ \\ ${ }^{1}$ Division of Organ Pathology, Department of Pathology, and ${ }^{2}$ Division of General Thoracic Surgery, \\ Department of Surgery, Faculty of Medicine, Tottori University, Tottori, Japan
}

\begin{abstract}
Background/Aim: Podoplanin is a candidate cancer stem cell marker in squamous cell carcinoma (SCC). Several studies have reported the prognostic value of podoplanin expression in tumor cells in lung SCC but few have focused on its expression in cancer-associated fibroblasts (CAFs). The aim of this study was to analyze the prognostic significance of podoplanin expression, with special reference to the expression pattern in both tumor cells and CAFs. Patients and Methods: Immunohistochemical analyses using anti-podoplanin antibody were performed on 126 resected specimens of lung SCC. When more than $10 \%$ of tumor cells or CAFs showed immunoreactivity with podoplanin levels as strong as those of the positive controls, the specimens were classified as a podoplanin-positive. Results: Podoplanin-positive status in tumor cells $(n=54)$ was correlated with a lower incidence of lymphatic invasion $(p=0.031)$ but there were no significant differences in diseasefree survival (DFS) and disease-specific survival (DSS) by the log-rank test. Podoplanin-positive status in CAFs $(n=41)$ was correlated with more advanced stage $(p=0.008)$, higher frequency of pleural invasion $(p=0.002)$ and both shorter DFS ( $p=0.006)$ and DSS ( $p=0.006)$. In Cox's multivariate analysis, podoplanin-positive status in CAFs was an independent negative prognostic factor for DFS $(p=0.027)$ and DSS ( $p=0.027)$. Conclusion: Podoplanin expression in
\end{abstract}

This article is freely accessible online.

Correspondence to: Yoshihisa Umekita, MD, Ph.D., Division of Organ Pathology, Department of Pathology, Faculty of Medicine, Tottori University, 86 Nishicho, Yonago, Tottori 683-8503, Japan. Tel: +81 859386053, Fax: +81 859386050, e-mail: yume@med.tottori-u.ac.jp

Key Words: Podoplanin, lung, squamous cell carcinoma, immunohistochemistry.
CAFs might be an independent unfavorable prognostic indicator in patients with lung SCC, irrespective of the expression status of tumor cells.

Lung cancer is the leading cause of cancer-related death around the world (1). The incidence of lung squamous cell carcinoma (SCC) has fallen over the past few decades; however, it remains a common type of lung cancer, accounting for more than 400,000 new cases worldwide each year $(1,2)$. The treatment options for SCC remain very limited, while outcomes of adenocarcinoma have improved because of recent advances in molecular targeted therapies (2). Therefore, a better understanding of how lung SCC progresses and improvement in the prognostic markers used to identify the patients most at risk for recurrences are necessary.

Podoplanin, a $38 \mathrm{kDa}$ type I transmembrane glycoprotein, was identified as a platelet aggregation-inducing factor in cancer cells (3), and has been shown to be a useful immunohistochemical marker of lymphatic vessels. Lymphatic vessel density examined using an antibody to podoplanin has been reported to be a prognostic factor in lung cancer (4-6). Podoplanin is also expressed in tumor cells in lung cancer (7-11), and is considered to regulate tumor progression and metastasis through various mechanisms, including epithelial-mesenchymal transition and platelet aggregation (12-14). Several previous studies reported that podoplanin expression in tumor cells in lung SCC was correlated with a lower incidence of lymph node metastasis and good prognosis (7-10), whereas another study reported that it was associated with poor prognosis (11). Highpodoplanin expression is also observed in stromal fibroblasts, or so-called cancer-associated fibroblasts (CAFs), in lung adenocarcinoma and SCC, which has been shown to be unfavorable prognostic factor $(15,16)$. Furthermore, a recent study found that podoplanin-positive fibroblasts enhanced tumor formation by a lung adenocarcinoma cell line (17). To 
our knowledge, only one report has mentioned the prognostic value of podoplanin expression in CAFs in patients with lung SCC (16). Moreover, as far as we are aware no study has focused on prognosis by subgrouping patients according to the expression pattern of podoplanin in both tumor cells and CAFs. The aim of the present study was, therefore, to clarify the prognostic value of podoplanin in patients with lung SCC by categorizing patients according to the podoplanin expression pattern in both tumor cells and CAFs.

\section{Patients and Methods}

Patients and tumor specimens. From January 2005 to December 2012 at the Tottori University Hospital, Japan, 146 consecutive patients who underwent surgical resection for the treatment of lung cancer diagnosed as primary lung SCC were enrolled in the present study. Twenty cases were excluded due to their operation being non-curative $(n=12$ cases: tumor invasion of the mediastinum or chest wall in five cases, severe lymph node metastasis in three, diagnostic wedge resection due to distant metastasis in two, dissemination in the thoracic cavity in one and residue of carcinoma in the bronchial stump in one case), unavailability of tumor specimen $(n=4)$, surgeryrelated death due to pneumonia $(n=2)$ and history of neoadjuvant treatment $(\mathrm{n}=2)$. Thus, 126 patients were included in this analysis. The median follow-up time was 48.0 months (range $=1-137$ months, mean=50.1 months). Patients' clinicopatho-logical data were retrieved from the hospital medical records. Pathological diagnosis was performed using hematoxylin and eosin-stained sections according to the criteria of the current World Health Organization classification for lung cancer (18). The following histopathological factors were evaluated: tumor size, tumor differentiation, lymph node metastasis, lymphatic invasion and pleural invasion. Lymphatic invasion was evaluated simultaneously when tumor podoplanin staining was assessed. Pleural invasion was evaluated by elastica van Gieson staining. Tumor stage was determined based on the seventh tumornode-metastasis (TNM) classification of lung cancer (19). Written informed consent was obtained and this study was conducted with the approval of the Ethics Committee of the Faculty of Medicine, Tottori University (approval number: 1606A028; June 23, 2016).

Immunohistochemistry. All resected specimens were fixed in $10 \%$ neutrally buffered formalin and embedded in paraffin. After the sections ( $4 \mu$ m-thick) were deparaffinized and endogenous peroxidase activity was blocked, they were pretreated in citrate buffer $(0.01 \mathrm{M}, \mathrm{pH} 6.0)$ at $98^{\circ} \mathrm{C}$ for $40 \mathrm{~min}$. After cooling to room temperature, nonspecific binding was blocked by blocking buffer (BLOCK ACE; Megmilk Snow Brand, Sapporo, Japan). The sections were then incubated at $4{ }^{\circ} \mathrm{C}$ overnight with an antibody to podoplanin (clone D2-40, diluted 1:100; Dako, Glostrup, Denmark). They were then incubated with the EnVision+ System HRP (Dako). Finally, the slides were incubated with diaminobenzidine (DAB) solution (liquid DAB+ substrate, imidazole-HCI buffer, $\mathrm{pH} 7.5$, containing hydrogen peroxide and an antimicrobial agent; Dako) for $4 \mathrm{~min}$, and counterstained with hematoxylin.

Evaluation of immunohistochemical findings. Regarding definition of immunoreactivity, lymphatic endothelium and type I pneumocytes were used as internal positive controls for podoplanin. When more than $10 \%$ of tumor cells or CAFs showed immunoreactivity with
Table I. Clinicopathological characteristics of 126 patients with lung squamous cell carcinoma.

\begin{tabular}{|c|c|c|}
\hline Factor & $\mathrm{N}$ & $(\%)$ \\
\hline Age, mean \pm SD (years) & \multicolumn{2}{|c|}{$73.9 \pm 8.25$} \\
\hline \multicolumn{3}{|l|}{ Gender } \\
\hline Male & 115 & $(91.3)$ \\
\hline Female & 11 & $(8.7)$ \\
\hline \multicolumn{3}{|l|}{ Smoking status } \\
\hline Ever smoker & 121 & $(96.0)$ \\
\hline Never smoker & 5 & $(4.0)$ \\
\hline \multicolumn{3}{|l|}{ Surgical procedure } \\
\hline Wedge resection & 32 & $(25.4)$ \\
\hline Segmentectomy & 13 & $(10.3)$ \\
\hline Lobectomy or more & 81 & $(64.3)$ \\
\hline \multicolumn{3}{|l|}{ Pathological T } \\
\hline $\mathrm{T} 1$ & 47 & $(37.3)$ \\
\hline $\mathrm{T} 2$ & 61 & (48.4) \\
\hline $\mathrm{T} 3$ & 16 & (12.7) \\
\hline $\mathrm{T} 4$ & 2 & (1.6) \\
\hline \multicolumn{3}{|l|}{ Pathological N } \\
\hline No & 95 & $(75.4)$ \\
\hline N1 & 26 & (20.6) \\
\hline $\mathrm{N} 2$ & 5 & $(4.0)$ \\
\hline \multicolumn{3}{|l|}{ Pathological stage } \\
\hline IA & 43 & $(34.1)$ \\
\hline IB & 36 & (28.6) \\
\hline IIA & 18 & (14.3) \\
\hline IIB & 18 & (14.3) \\
\hline IIIA & 11 & $(8.7)$ \\
\hline \multicolumn{3}{|l|}{ Lymphatic invasion } \\
\hline Absent & 90 & (71.4) \\
\hline Present & 36 & (28.6) \\
\hline \multicolumn{3}{|l|}{ Pleural invasion } \\
\hline Absent & 82 & $(65.1)$ \\
\hline Present & 44 & (34.9) \\
\hline
\end{tabular}

podoplanin as strong as that of the positive controls, the specimens were classified as a podoplanin-positive. CAFs were defined as the spindle-shaped cells present in tumor stroma. All slides were evaluated by Y.Y. and Y.U. who were blinded to the patients' clinicopathological data.

Statistical analysis. All statistical analyses were performed with SPSS version 23 (IBM SPSS Statistics; IBM Corporation, Armonk, NY, USA). The association between clinicopathological factors and podoplanin expression status was evaluated by the chi-square test. For survival analyses, two different endpoints, cancer relapse and cancerrelated death, were used to calculate disease-free survival (DFS) and disease-specific survival (DSS), respectively. DFS was defined as the period from the date of primary surgery to the date of clinical or pathological cancer relapse. DSS was defined as a period from the date of primary surgery to the date of cancer-related death. In the calculation of DFS and DSS, patients were censored at the time of their last cancer-free clinical follow-up or at the time of death if it was not related to cancer. Survival curves were plotted by the KaplanMeier method and differences in DFS and DSS were analyzed using the log-rank test. The Cox regression hazard model was used to 
A

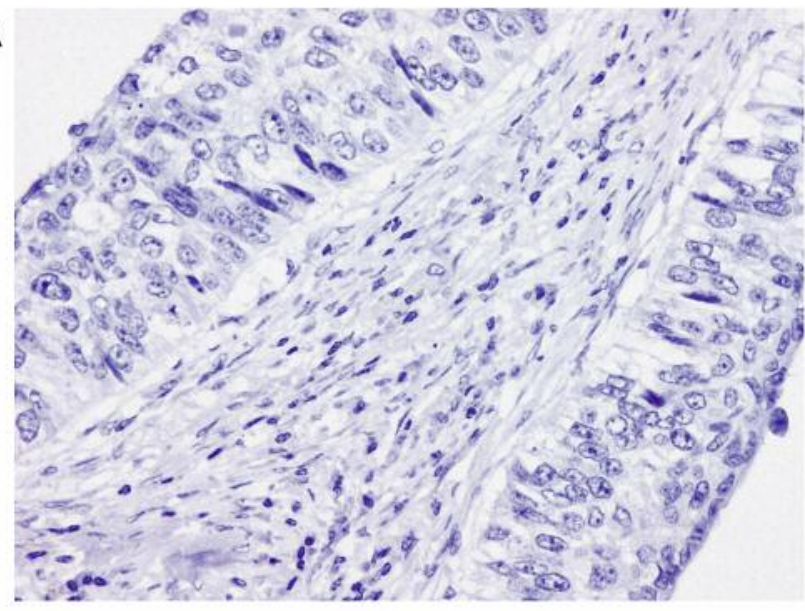

B
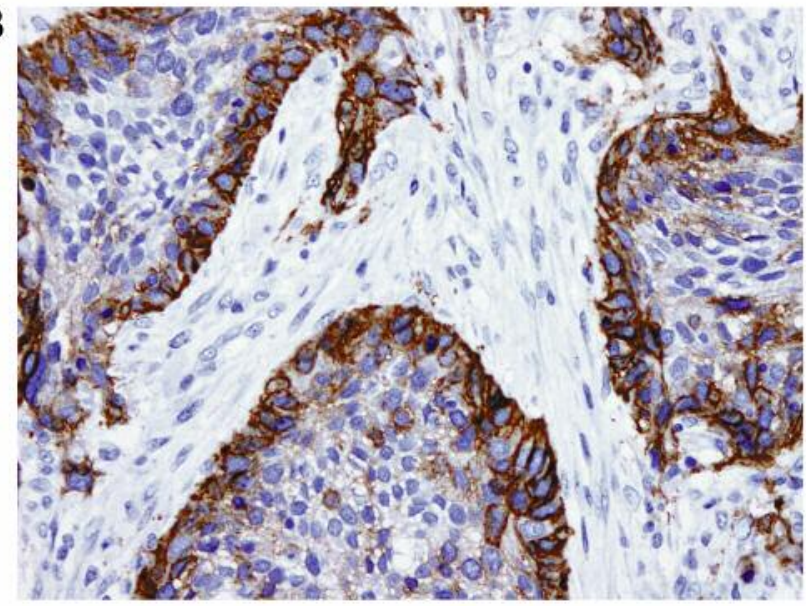
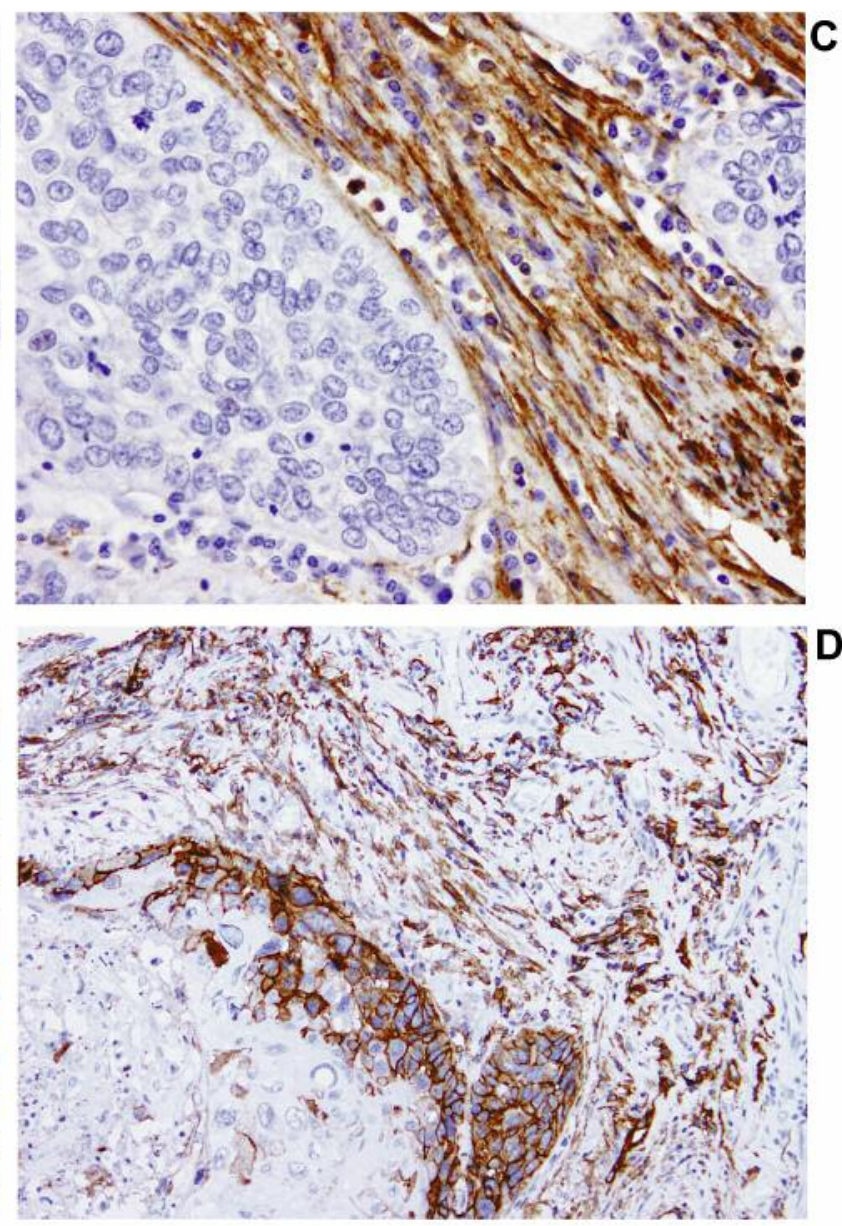

Figure 1. Immunohistochemical staining patterns of podoplanin in lung squamous cell carcinoma. A: Negative expression in both tumor cells and cancer-associated fibroblasts (CAFs). B: Podopolanin-positive expression in tumor cells and-negative expression in CAFs. C: Podoplanin-negative expression in tumor cells and-positive expression in CAFs. D: Podoplanin-positive expression in both tumor cells and CAFs.

perform univariate and multivariate analysis for prognostic factors for DFS and DSS. All tests were two-sided, and $p$-values less than 0.05 were considered to be statistically significant in all tests.

\section{Results}

Immunohistochemistry. Representative immunohistochemical staining patterns of podoplanin are shown in Figure 1. Immunoreactivity for tumor cells was observed mainly on the cell membrane and was predominantly found in the peripheral layers of tumor nests (Figure 1B and D). The podoplanin expression in tumor cells and CAFs in lung SCC exhibited four main staining patterns (Figure 1).

Clinicopathological characteristics and association with podoplanin expression status. The clinicopathological characteristics of the 126 patients with SCC are summarized in Table I. The mean age of the patients was $73.9 \pm 8.25$ years. Almost all patients were males and ever-smokers. Of the 126 patients, $79(62.7 \%)$ had stage I disease, 36 (28.6\%) stage II, and $11(8.7 \%)$ stage III.

The associations between the clinicopathological characteristics and podoplanin expression status in tumor cells and CAFs of 126 patients are shown in Table II. Podoplanin-positive tumor cells were correlated with a lower incidence of lymphatic invasion $(p=0.031)$, whereas podoplanin-positive CAFs were correlated with a more advanced stage $(p=0.008)$ and a higher frequency of pleural invasion $(p=0.002)$.

Survival analysis according to podoplanin expression status. For the survival analysis, we first divided the 126 patients with lung SCC into four groups of patients who were podoplanin-negative for both tumor cells and CAFs (T-/C-), 

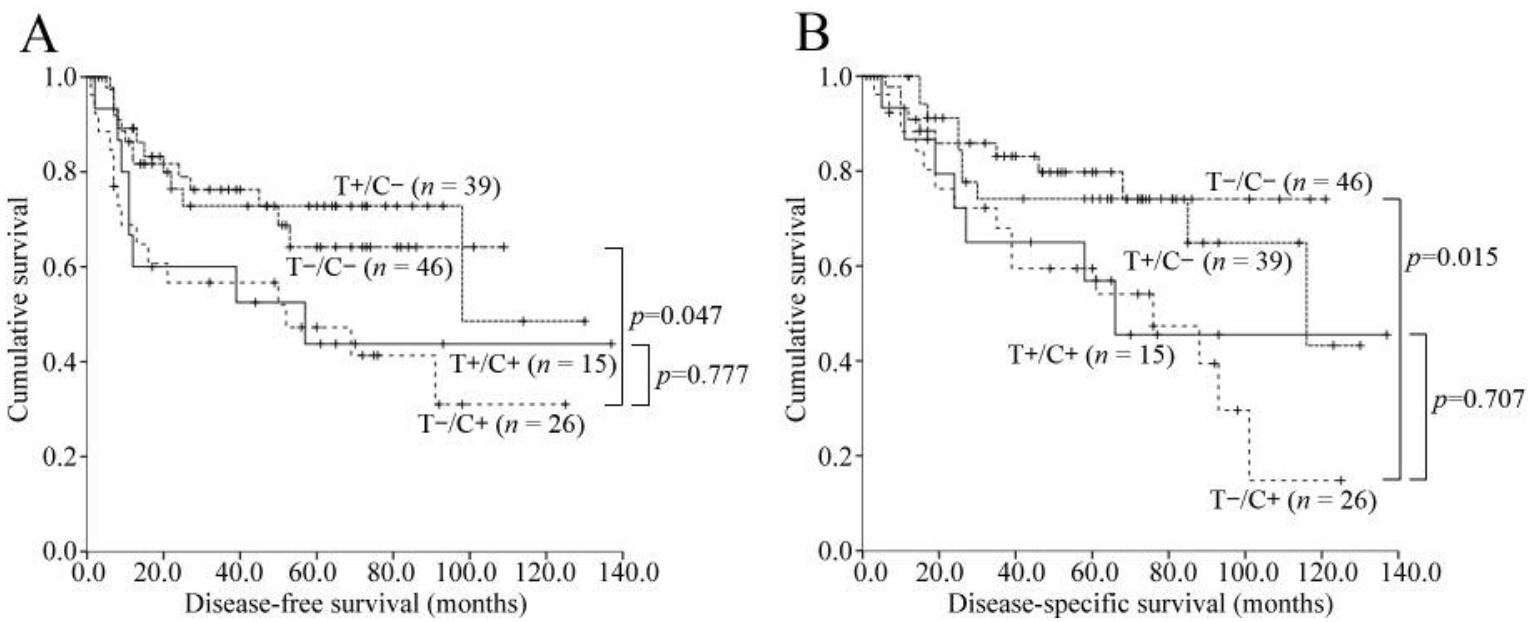

Figure 2. Kaplan-Meier survival curves for disease-free (A) and disease-specific (B) survival of 126 patients according to four podoplanin expression patterns: podoplanin-negative for both tumor cells and CAFs (T-/C-), popodoplanin-positive for tumor cells and -negative for CAFs (T+/C-), podoplanin-negative for tumor cells and -positive for CAFs $(T-/ C+)$, and podoplanin-positive for both tumor cells and CAFs $(T+/ C+)$.

Table II. Association between clinicopathological characteristics and podoplanin expression.

\begin{tabular}{|c|c|c|c|c|c|c|}
\hline \multirow{3}{*}{ Factors } & \multicolumn{6}{|c|}{ Podoplanin expression, no. of patients } \\
\hline & \multicolumn{3}{|c|}{ Cancer cells } & \multicolumn{3}{|c|}{ CAFs } \\
\hline & Negative $(N=72)$ & Positive $(\mathrm{N}=54)$ & $p$-Value & Negative $(\mathrm{N}=85)$ & Positive $(\mathrm{N}=41)$ & $p$-Value \\
\hline \multicolumn{7}{|l|}{ Gender } \\
\hline Male & 67 & 48 & 0.412 & 77 & 38 & 0.696 \\
\hline Female & 5 & 6 & & 8 & 3 & \\
\hline \multicolumn{7}{|l|}{ Age } \\
\hline$<75$ Years & 30 & 31 & 0.08 & 38 & 15 & 0.387 \\
\hline$\geq 75$ Years & 42 & 23 & & 47 & 26 & \\
\hline \multicolumn{7}{|l|}{ Smoking status } \\
\hline Ever smoker & 70 & 51 & 0.429 & 82 & 39 & 0.716 \\
\hline Never smoker & 2 & 3 & & 3 & 2 & \\
\hline \multicolumn{7}{|l|}{ Surgery } \\
\hline Lobectomy or more & 47 & 34 & 0.788 & 51 & 30 & 0.148 \\
\hline Seg/wedge & 25 & 20 & & 34 & 11 & \\
\hline \multicolumn{7}{|l|}{ Tumor size } \\
\hline$\leq 3 \mathrm{~cm}$ & 29 & 27 & 0.277 & 42 & 14 & 0.106 \\
\hline$>3 \mathrm{~cm}$ & 43 & 27 & & 43 & 27 & \\
\hline \multicolumn{7}{|l|}{ Lymph node metastasis } \\
\hline Absent & 52 & 43 & 0.339 & 65 & 30 & 0.687 \\
\hline Present & 20 & 11 & & 20 & 11 & \\
\hline \multicolumn{7}{|l|}{ Stage } \\
\hline I & 43 & 36 & 0.425 & 60 & 19 & 0.008 \\
\hline II/III & 29 & 18 & & 25 & 22 & \\
\hline \multicolumn{7}{|l|}{ Lymphatic invasion } \\
\hline Absent & 46 & 44 & 0.031 & 63 & 15 & 0.660 \\
\hline Present & 26 & 10 & & 22 & 4 & \\
\hline \multicolumn{7}{|l|}{ Pleural invasion } \\
\hline Absent & 45 & 37 & 0.483 & 63 & 19 & 0.002 \\
\hline Present & 27 & 17 & & 22 & 22 & \\
\hline
\end{tabular}

CAFs: Cancer-associated fibroblasts. 
A

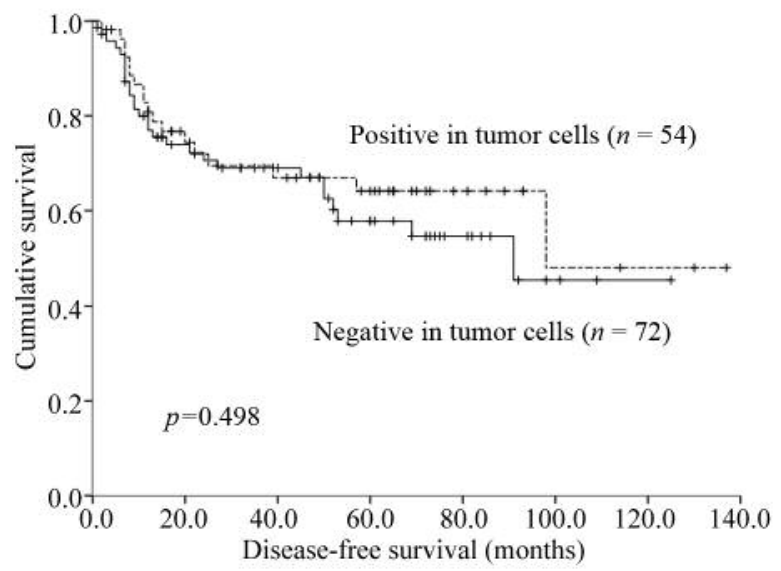

B

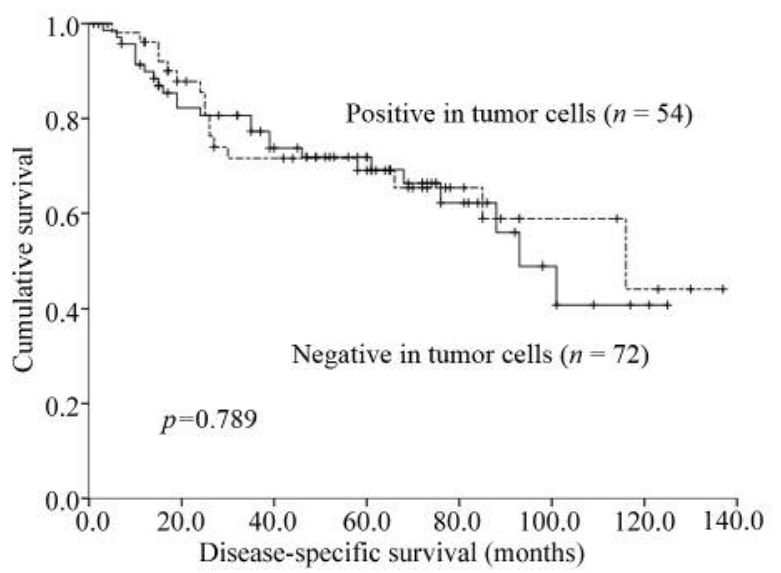

Figure 3. Kaplan-Meier survival curves for disease-free (A) and disease-specific (B) survival of 126 patients according to podoplanin expression status of tumor cells. There were 54 patients in the positive group and 72 patients in the negative group.

A

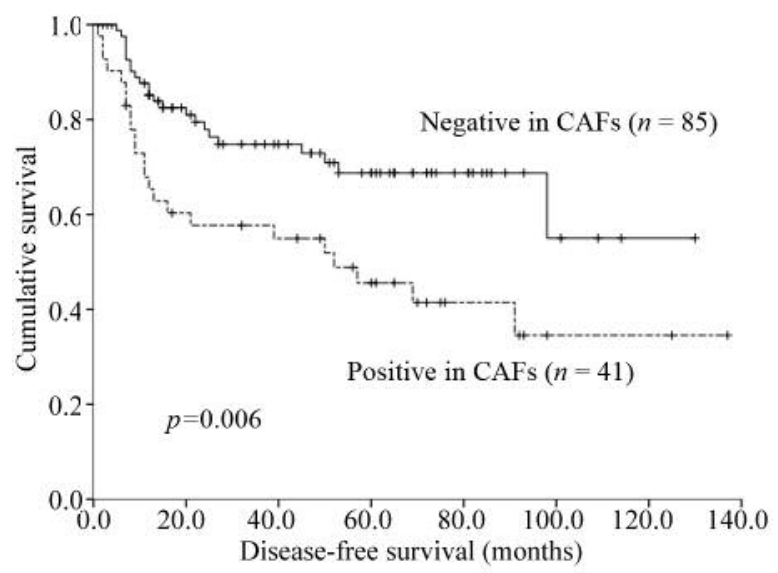

B

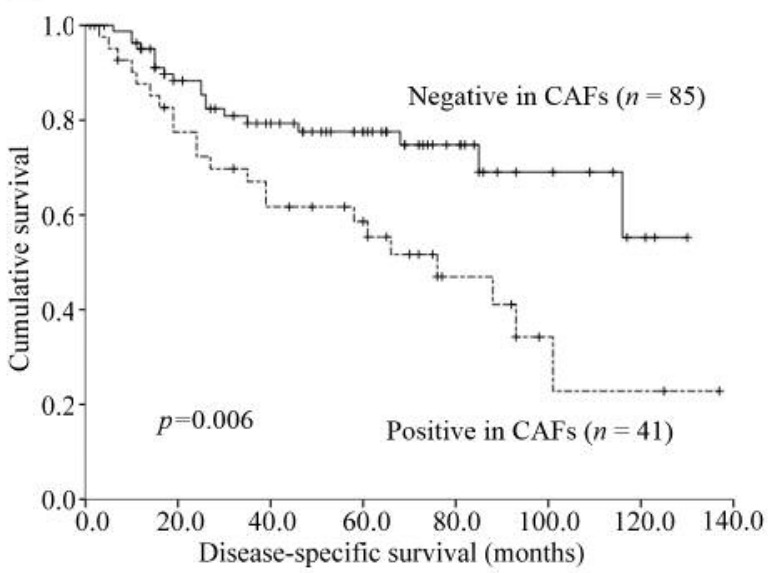

Figure 4. Kaplan-Meier survival curves for disease-free (A) and disease-specific (B) survival of 126 patients according to podoplanin expression status in cancer-associated fibroblasts $(C A F s)$. There were 41 patients in the positive group and 85 patients in the negative group.

podoplanin-positive for tumor cells and -negative for CAFs $(\mathrm{T}+/ \mathrm{C}-)$, podoplanin-negative for tumor cells and -positive for CAFs $(\mathrm{T}-/ \mathrm{C}+)$ and podoplanin-positive for both tumor cells and $\mathrm{CAFs}(\mathrm{T}+/ \mathrm{C}+)$. According to the survival curves of these four subgroups, there was a significant difference only between $\mathrm{T}-/ \mathrm{C}-$ and $\mathrm{T}-/ \mathrm{C}+$ according to the log-rank test in DFS $(p=0.047)$ and DSS $(p=0.015)$, while there were no differences between $\mathrm{T}+/ \mathrm{C}+$ and $\mathrm{T}-/ \mathrm{C}+$ in DFS $(p=0.777)$ and DSS $(p=0.707)$ (Figure 2). Next, we separately analyzed podoplanin expression in tumor cells or CAFs. Podoplanin-positive and -negative tumor cells were observed in 54 and 72 patients, respectively. According to the log-rank test, there were no significant differences between the two groups in DFS $(p=0.498)$ nor DSS $(p=0.798)$ (Figure 3). Podoplanin-positive CAFs were observed in 41 and 85 patients, respectively. The 5-year DFS rate of podoplanin-positive and negative groups were $45.6 \%$ [95\% confidence interval $(\mathrm{CI})=29.5-61.7 \%$ ] and $68.7 \%$ (95\% CI=57.5-80.0\%), respectively. The 5-year DSS rates of the two groups were $58.6 \%$ (95\% CI=42.9$74.3 \%$ ) and $77.6 \%$ (95\% CI=67.8-87.4\%), respectively. The log-rank test revealed that the podoplanin-positive group had significantly shorter DFS $(p=0.006)$ and DSS $(p=0.006)$ (Figure 4). Multivariate analyses using the Cox 
Table III. Multivariate analyses of various prognostic factors in 126 patients with lung squamous cell carcinoma.

\begin{tabular}{|c|c|c|c|c|c|c|}
\hline \multirow[t]{2}{*}{ Prognostic factor } & \multicolumn{3}{|c|}{ Disease-free survival } & \multicolumn{3}{|c|}{ Disease-specific survival } \\
\hline & HR & $95 \% \mathrm{CI}$ & $p$-Value & HR & $95 \% \mathrm{CI}$ & $p$-Value \\
\hline Sex: Male vs. female & 1.133 & $0.337-3.815$ & 0.840 & 0.903 & $0.264-3.084$ & 0.871 \\
\hline Age: $\geq 75 v s .<75$ years & 0.692 & $0.375-1.277$ & 0.239 & 0.786 & $0.410-1.506$ & 0.468 \\
\hline Tumor size: $>3 v s . \leq 3 \mathrm{~cm}$ & 1.103 & $0.557-2.184$ & 0.778 & 1.069 & $0.504-2.264$ & 0.863 \\
\hline Lymph node metastasis: present $v s$. absent & 1.030 & $0.509-2.081$ & 0.935 & 1.112 & $0.530-2.330$ & 0.779 \\
\hline Lymphatic invasion: present $v s$. absent & 1.331 & $0.688-2.574$ & 0.395 & 1.397 & $0.702-2.780$ & 0.341 \\
\hline Pleural invasion: present $v s$. absent & 1.157 & $0.573-2.337$ & 0.684 & 1.214 & $0.583-2.526$ & 0.605 \\
\hline Podoplanin in CAFs: positive vs. negative & 2.075 & $1.088-3.957$ & 0.027 & 2.170 & $1.091-4.319$ & 0.027 \\
\hline
\end{tabular}

HR: Hazard ratio; CI: confidence interval; CAFs: cancer-associated fibroblasts.

regression hazard model showed that being podoplaninpositive in CAFs was the only independent prognostic factor for shorter DFS $(p=0.027)$ and DSS $(p=0.027)$ in 126 patients with lung SCC (Table III).

\section{Discussion}

Many studies have investigated the prognostic factors for non-small cell lung cancer or lung adenocarcinoma; however, there have been relatively few reports with regard to SCC. The pathogenesis and biological characteristics of SCC differ from those of adenocarcinoma (2), therefore, it may be desirable to explore the prognostic factors specific to patients with SCC. Podoplanin expression has been widely used for the detection of lymphatic vessel invasion in many cancer types. Its expression rate in lung SCC cells is higher than that in adenocarcinoma of the lung (11), and podoplanin has been considered as a candidate stem cell marker in SCC (20). Collectively, these findings led us to speculate that podoplanin may be a candidate prognostic marker for lung SCC. Indeed, several studies have reported that podoplanin expression in tumor cells had a prognostic impact for patients with SCC; however, there are conflicting results regarding its favorability (7-10) or unfavorability (11) as a prognostic indicator. A large series that included 136 patients with pathological stage IB SCC of the lung revealed a significant correlation between podoplanin negativity and poor outcome in patients aged 70 years or more (8). In contrast, Kadota et al. reported that podoplaninpositive status was a poor prognostic factor for patients with SCC of the lung (11). In the present study, we used the same cut-off value $(10 \%)$ as the previous studies $(8-10,16)$ but failed to demonstrate a significant correlation between podoplanin expression in tumor cells and prognosis. Although other cut-off values such as $1 \%$ or $50 \%$ were also used in our study, no significant differences between podoplanin expression in tumor cells and prognosis were observed (data not shown). Factors contributing to this complexity may include the differences in the positive criteria, study population, methods of detection etc.

Recently, several cell culture studies have suggested that podoplanin promoted epithelial-mesenchymal transition (12, 13), and that podoplanin in carcinoma cells induced platelet aggregation and promoted pulmonary metastasis in animal models (14). Thus, further investigation of the function of podoplanin in pulmonary SCC is needed to validate the role of podoplanin in carcinoma cells and its prognostic significance. In contrast, to our knowledge, there have been very few reports focusing on podoplanin expression in CAFs as a prognostic factor in patients with lung cancer $(15,16)$. Ono et al. reported that high podoplanin expression in CAFs and low E-cadherin expression in cancer cells were associated with significantly worse prognosis in stage I lung SCC (16). Kawase et al. revealed that high podoplanin expression in CAFs predicted poor prognosis in lung adenocarcinoma (15), however; it was not an independent prognostic factor according to multivariate analysis.

As far as we know, ours is the first report focusing on the prognostic relevance of podoplanin expression in both tumor cells and CAFs in patients with lung SCC. Regarding the role of podoplanin in fibroblasts, it has been reported that human vascular adventitial fibroblasts that highly expressed podoplanin enhanced tumor formation in lung adenocarcinoma cell lines more than human lung tissue-derived fibroblasts that expressed podoplanin at a lower level (17). These results suggest that the tumor microenvironment created by podoplanin-positive CAFs promotes tumor progression and the acquisition of an aggressive character. In the present study, we found no significant differences in either DFS or DSS between the $\mathrm{T}+/ \mathrm{C}+$ and $\mathrm{T}-/ \mathrm{C}+$ subgroups; however, no definitive conclusion was drawn because the number of samples was too small. A larger study is required to reveal any prognostic difference between the $\mathrm{T}+/ \mathrm{C}+$ and $\mathrm{T}-/ \mathrm{C}+$ subgroups and thereby gain a better understanding of the interaction between tumor cell and CAFs. 
In conclusion, for the first time to our knowledge, we revealed that podoplanin expression in CAFs was an independent indicator of worse prognosis, irrespective of the expression status of the tumor cells in patients with lung SCC. The limitations of our study were the small number of patients and its retrospective nature. Although further studies with a larger series of patients and a longer follow-up period are required, our results suggested that immunohistochmical analysis using antibodies to podoplanin, which has been routinely performed, could be useful not only for the detection of lymphatic vessel invasion but also for predicting an aggressive tumor phenotype in patients with lung SCC.

\section{Acknowledgements}

The Authors thank Kazuo Yashima and Kazuko Fukushima for their excellent technical assistance with the processing of the pathological specimens.

\section{References}

1 Torre LA, Bray F, Siegel RL, Ferlay J, Lortet-Tieulent J and Jemal A: Global cancer statistics, 2012. CA Cancer J Clin 65(2): 87-108, 2015.

2 Sereno M, Esteban IR, Zambrana F, Merino M, Gómez-Raposo C, López-Gómez M and Sáenz EC: Squamous-cell carcinoma of the lungs: is it really so different? Crit Rev Oncol Hematol 84(3): 327-339, 2012.

3 Kaneko MK, Kato Y, Kitano T and Osawa M: Conservation of a platelet activating domain of Aggrus/podoplanin as a platelet aggregation-inducing factor. Gene 378: 52-57, 2006.

4 Renyi-Vamos F, Tovari J, Fillinger J, Timar J, Paku S, Kenessey I, Ostoros G, Agocs L, Soltesz I and Dome B: Lymphangiogenesis correlates with lymph node metastasis, prognosis, and angiogenic phenotype in human non-small cell lung cancer. Clin Cancer Res 11(20): 7344-7353, 2005.

5 Kadota K, Huang CL, Liu D, Ueno M, Kushida Y, Haba R and Yokomise $\mathrm{H}$ : The clinical significance of lymphangiogenesis and angiogenesis in non-small cell lung cancer patients. Eur J Cancer 44(7): 1057-1067, 2008.

6 Iwakiri S, Nagai S, Katakura H, Takenaka K, Date H, Wada H and Tanaka F: D2-40-positive lymphatic vessel density is a poor prognostic factor in squamous cell carcinoma of the lung. Ann Surg Oncol 16(6): 1678-1685, 2009.

7 Suzuki H, Onimaru M, Koga T, Takeshita M, Yano T, Maehara $\mathrm{Y}$, Nakamura $\mathrm{S}$ and Sueishi K: High podoplanin expression in cancer cells predicts lower incidence of nodal metastasis in patients with lung squamous cell carcinoma. Pathol Res Pract 207(2): 111-115, 2011

8 Ito T, Ishii G, Nagai K, Nagano T, Kojika M, Murata Y, Atsumi N, Nishiwaki Y, Miyazaki E, Kumamoto T and Ochiai A: Low podoplanin expression of tumor cells predicts poor prognosis in pathological stage IB squamous cell carcinoma of the lung, tissue microarray analysis of 136 patients using 24 antibodies. Lung Cancer 63(3): 418-424, 2009.
9 Shimada Y, Ishii G, Nagai K, Atsumi N, Fujii S, Yamada A, Yamane Y, Hishida T, Nishimura M, Yoshida J, Ikeda N and Ochiai A: Expression of podoplanin, CD44, and p63 in squamous cell carcinoma of the lung. Cancer Sci 100(11): 2054-2059, 2009.

10 Ikoma Y, Kijima H, Masuda R, Tanaka M, Inokuchi S and Iwazaki M: Podoplanin expression is correlated with the prognosis of lung squamous cell carcinoma. Biomed Res 36(6): 393-402, 2015.

11 Kadota K, Huang CL, Liu D, Nakashima N, Yokomise H, Ueno $\mathrm{M}$ and Haba R: The clinical significance of tumor cell D2-40 immunoreactivity in non-small cell lung cancer. Lung Cancer 70(1): 88-93, 2010.

12 Martín-Villar E, Megías D, Castel S, Yurrita MM, Vilaró S and Quintanilla M: Podoplanin binds ERM proteins to activate RhoA and promote epithelial-mesenchymal transition. J Cell Sci 119(Pt 21): 4541-4553, 2006.

13 Fernández-Muñoz B, Yurrita MM, Martín-Villar E, CarrascoRamírez P, Megías D, Renart J and Quintanilla M: The transmembrane domain of podoplanin is required for its association with lipid rafts and the induction of epithelial-mesenchymal transition. Int J Biochem Cell Biol 43(6): 886-896, 2011.

14 Kunita A, Kashima TG, Morishita Y, Fukayama M, Kato Y, Tsuruo T and Fujita N: The platelet aggregation-inducing factor aggrus/podoplanin promotes pulmonary metastasis. Am J Pathol 170(4): 1337-1347, 2007.

15 Kawase A, Ishii G, Nagai K, Ito T, Nagano T, Murata Y, Hishida T, Nishimura M, Yoshida J, Suzuki K and Ochiai A: Podoplanin expression by cancer associated fibroblasts predicts poor prognosis of lung adenocarcinoma. Int J Cancer 123(5): 10531059, 2008.

16 Ono S, Ishii G, Nagai K, Takuwa T, Yoshida J, Nishimura M, Hishida T, Aokage K, Fujii S, Ikeda $\mathrm{N}$ and Ochiai A: Podoplanin-positive cancer-associated fibroblasts could have prognostic value independent of cancer cell phenotype in stage I lung squamous cell carcinoma: usefulness of combining analysis of both cancer cell phenotype and cancer-associated fibroblast phenotype. Chest 143(4): 963-970, 2013.

17 Hoshino A, Ishii G, Ito T, Aoyagi K, Ohtaki Y, Nagai K, Sasaki H and Ochiai A: Podoplanin-positive fibroblasts enhance lung adenocarcinoma tumor formation: podoplanin in fibroblast functions for tumor progression. Cancer Res 71(14): 4769-4779, 2011.

18 Travis WD, Brambilla E, Burke AP, Marx A and Nicholson AG: WHO Classification of Tumours of the Lung, Pleura, Thymus and Heart, Fourth Edition. IARC, Lyon, 2015.

19 Goldstraw P, Crowley J, Chansky K, Giroux DJ, Groome PA, Rami-Porta R, Postmus PE, Rusch V, Sobin L: The IASLC Lung Cancer Staging Project: proposals for the revision of the TNM stage groupings in the forthcoming (seventh) edition of the TNM Classification of Malignant Tumors. J Thorac Oncol 2(8): 706$714,2007$.

20 Atsumi N, Ishii G, Kojima M, Sanaba M, Fujii S and Ochiai A: Podoplanin a novel marker of tumor-initiating cells in human squamous cell carcinoma A431. Biochem Biophys Res Commun 373: 36-41, 2008.

Received November 4, 2016

Revised November 24, 2016

Accepted November 29, 2016 